\title{
A Novel Ultrasound Imaging Mobile Connectivity App
}

\author{
George $\mathrm{Ku}$ \\ Staff Software Engineer \\ Siemens Medical Solutions, USA, Inc. \\ Plymouth Meeting, PA USA
}

\author{
Jared Livingston \\ Senior User Experience Engineer \\ IdentityMine \\ Seattle, WA, USA
}

\author{
Jeffrey Blankenburg \\ Senior Program Manager \\ Microsoft Corporation \\ Redmond, WA, USA
}

\begin{abstract}
ACUSON Freestyle MobileLink app is a Microsoft ${ }^{\circledR}$ Windows ${ }^{\circledR}$ mobile app which communicates with the Siemens ACUSON Freestyle ${ }^{\mathrm{TM}}$ ultrasound system. The app enables the user to review and share saved ultrasound studies from the ACUSON Freestyle system. It also provides the ability to add patient demographic information to the system worklist. The app runs on a Microsoft Windows tablet or mobile device, using Windows 8.1 or higher.
\end{abstract}

\section{INTRODUCTION}

The ACUSON Freestyle system (Figure 1) is a portable ultrasound system manufactured and marketed by Siemens Medical Solutions USA, Inc. The ACUSON Freestyle system is the world's first ultrasound system with wireless transducers. With outstanding image quality and ease-of-use, the system is designed to advance the use of ultrasound as a tool in guiding interventional procedures.

The advantages of a wireless transducer include: simpler preparation for sterile procedures, greater flexibility in positioning the system and the transducer, and freedom from cable weight or torque for greater ease in obtaining accurate transducer and needle positioning [1].

The ACUSON Freestyle system engineering team, in collaboration with Microsoft Corporation and IdentityMine, has developed a Microsoft Windows app to link the system with Windows-based mobile devices, such as a Microsoft Surface ${ }^{\mathrm{TM}}$ tablet.

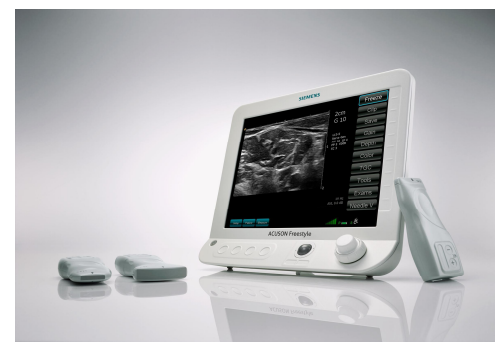

Figure 1. Siemens ACUSON Freestyle ${ }^{\mathrm{TM}}$ system main unit and probe.

\section{SYSTEM ARCHITECTURE}

The ACUSON Freestyle system main unit contains the backend processing system with LCD panel. The user interface consists of a trackball, rotary knobs, and capacitive sense buttons. It utilizes a digital media processor as its Central Processing Unit (CPU), running an embedded operating system.

The ultrasound system communicates with the probe using two communications channels, 1) Lossless digital image data high speed wireless transmission using a proprietary 7.8 GHz ultra-wideband radio, 2) Backchannel Bluetooth ${ }^{\circledR}$ radio used for bidirectional control and data communication [2].

The ultrasound system has both wireless and Ethernet network connections. The network connection is used to communicate with the ACUSON Freestyle MobileLink app. It can also be used to communicate with networked DICOM (Digital Imaging and Communications in Medicine) [3] systems for the archiving of images and obtaining worklist data (list of patient demographic data for studies to be performed).

Patient study information and the related study images are stored by the CPU in flash memory. The system maintains a list of studies currently present on the system in an XML file. This includes an identifier string that can be used to locate the study data in flash memory.

Patient study data for individual studies is stored in the system in XML files. This data includes patient demographic information for each study. This information can be either manually entered into the system or received from the DICOM network. Images acquired during the study are stored as JPG images or QuickTime ${ }^{\circledR}$ movies [4]. The Motion-JPEG format is used for movies. The system can export studies in both native formats (JPG, MOV and XML files) to a USB drive, in DICOM format to a USB drive or to a networked DICOM image archiving system. For DICOM 
exports, the system converts the stored JPG and MOV files to DICOM format.

\section{APP CONCEPT}

The original concept of the app was to provide a method for ACUSON Freestyle system users to contact Siemens support personnel with questions, for advice, or to request a service call via email. In many cases, attaching an image will often illustrate the issue for which the customer seeks advice. Additionally, such an app could automatically supply basic system information such as serial number and software version, rather than having the customer locate and record this information.

Using these general ideas and drawing on the system's use of XML files and images stored in standard formats, the app concept was further developed into a method to pull entire studies onto a mobile device, display the images, and email selected images. The mobile device's touch interface: tapping to select, swiping to navigate between images and stretch/pinch gestures to enlarge/shrink images can be used.

Communication between the mobile device and the ACUSON Freestyle system is done over the wireless / Ethernet interfaces of the devices. A TCP/IP socket implementation is used, and a separate execution thread would be implemented on the ultrasound system to handle communication with the mobile device.

A TCP/IP connection between the app and the ACUSON Freestyle system is initiated by user action on the mobile device. In response, the ultrasound system transfers data (typically XML, image, or movie files) to the mobile device. Once the entire dataset is received, the TCP/IP connection is released by the mobile app.

The system's XML files can easily be parsed by the mobile app to obtain study information. Since the images saved on the ACUSON Freestyle system are in standard formats, image and movie viewers that are native to the mobile device can be used in the app without modification.

\section{APP DEVELOPMENT}

The ACUSON Freestyle MobileLink App was developed by IdentityMine in Seattle, Washington. IdentityMine is an interactive and user experience (UX) development company specializing in natural user interfaces (NUI). IdentityMine blends innovative design with expertise in: Windows Phone, Windows 8.1, Kinect for Windows, Xbox 360 and Xbox One, SmartGlass, HTML5, WPF, Xamarin and others.

\footnotetext{
IdentityMine led the design and architecture of the
N Freestyle

IdentityMine led the design and architecture of the
ACUSON Freestyle the Siemens team. The design of the application targeted
}

different resolutions and device form factors. The IdentityMine team approached the development with a layered architecture to separate the user interface (UI) from business logic, the data model and service access layers. Using Visual Studio 2013 in conjunction with Expression Blend, the application was written using the programming language $\mathrm{C \#}$ and the mark-up language XAML [5]. To take advantage of the full potential of XAML, IdentityMine implemented the MVVM (Model-View-ViewModel) pattern [6] as the architectural base for the application. To ensure effective team collaboration and production of a quality product, IdentityMine adhered to the strict separation of UI, data models and business logic. In accordance with the MVVM pattern, the View layer was written completely in XAML and it has a loose dependency on the business logic contained in the C\# ViewModels. IdentityMine implemented the use of asynchronous socket communication to facilitate communication with the ACUSON Freestyle ${ }^{\mathrm{TM}}$ ultrasound system. Using the asynchronous method calls feature in the Windows 8 platform allows the application to remain responsive while long-running data retrieval methods are executing.

The app will be available on the Microsoft Windows Store in the fourth quarter of calendar year 2014. The app will be free.

\section{StUdy DisPlay COMMANDS}

A very simple set of commands is used to implement the basic functionality of pulling and displaying studies from the ultrasound system.

The "REGISTER" command is sent from the mobile device and is used to establish communication to the ultrasound system. This command includes a PIN code which must match the ACUSON Freestyle MobileLink PIN code set on the ultrasound system. If the PIN codes match, the ACUSON Freestyle system generates a Globally Unique ID (GUID) string which is sent back to the mobile device with the system serial number and software version. The GUID string is sent from the app with each subsequent command and is verified by the ACUSON Freestyle before command execution.

A "STUDYLIST" command is sent from the mobile device to obtain the list of studies stored on the system. The system, in response to this command, sends an XML file to the mobile device. This file contains the patient name, patient ID, study date / time, and identifying / locating information for each of the studies. The list of studies is displayed on the mobile device (Figure 2). 


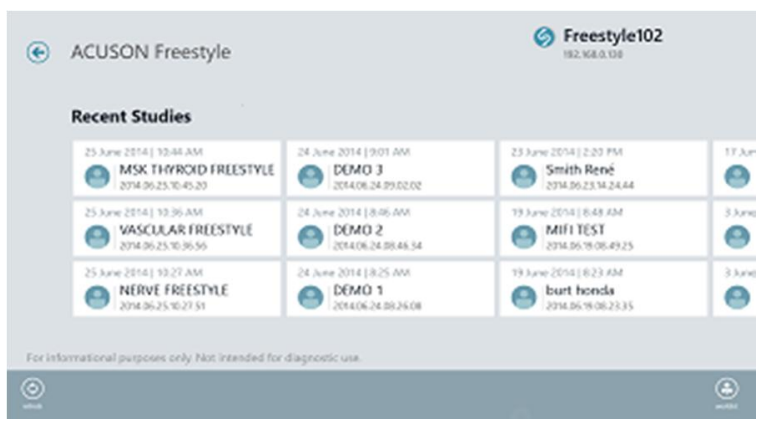

Figure 2. Study List Screen

To view a study, the user of the mobile device selects a study by tapping a study on the list. The mobile device sends an "ALLIMAGES" command with the selected study's identifying information. The system then responds by sending the filename and image data for all of the saved images. A thumbnail view of the entire study is displayed (Figure 3).

Still images are differentiated from movie files by the filename suffix (JPG or MOV). For still images, the complete JPG file is sent. For movie images, only the first frame of the movie is sent. No study-related files are permanently stored on the mobile device.

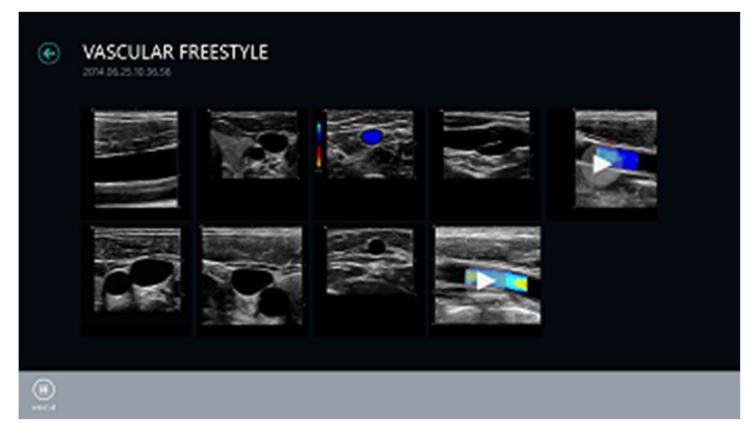

Figure 3. Study thumbnail view

On the mobile device, movie files are displayed with a play button centered on the image. Once pressed, a "GETDOCUMENT" command is issued to the system, along with the desired filename. The system responds to this command by sending the entire movie to the mobile device to be played.

Once a thumbnail image is tapped, the image or movie is displayed to fill the screen, while maintaining the image's aspect ratio (Figure 4). Images and movies can be enlarged or shrunk by using stretching or pinching gestures. The study can be navigated by swiping through the images.

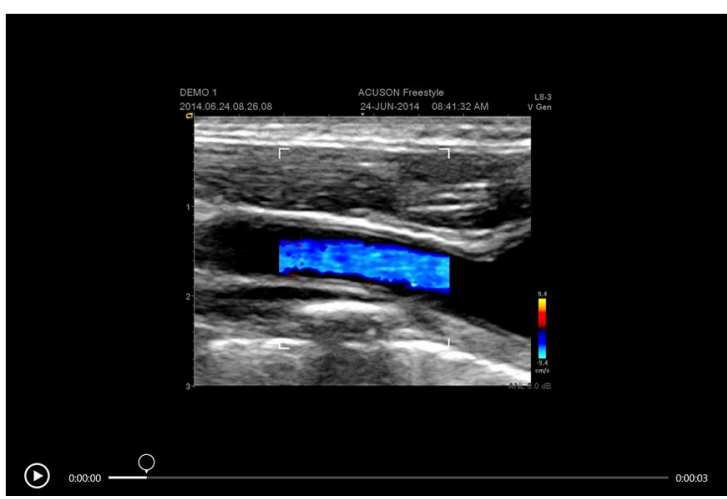

Figure 4. Image View, showing movie controls

\section{Sharing Of IMAGes}

Selected images can be shared using the features of the mobile device's operating system. Sharing of an image or movie by email automatically includes the system's serial number and software version. The statement "For informational purposes only. Not intended for diagnostic use." is also included in the body of the email.

\section{AdDING To ULTRASOUND WORKLIST}

The ACUSON Freestyle system has the ability to query a hospital information system to obtain a list of patients (a worklist) that are scheduled to be scanned. This function is called a DICOM Modality Worklist query.

The worklist contains the patient demographic information for each patient. When the patient arrives for the exam, he/she is selected from the ultrasound system's worklist, automatically populating the demographic information into the patient data entry screen. This feature eliminates the potentially time-consuming process of manual patient registration on the imaging system.

The ACUSON Freestyle system provides an interface for the app to add to or create a worklist. The app has a screen that allows the entry of basic patient demographic information: patient name, ID, gender, date of birth and accession number (Figure 5). This information is sent to the system using the "WORKLIST" command.

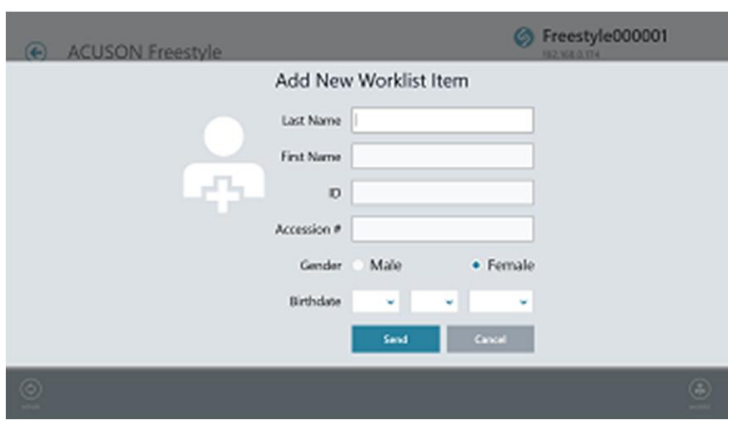

Figure 5. Worklist entry screen 


\section{SECURITY}

The system and the app have measures to prevent unauthorized access to saved studies. A user of the ACUSON Freestyle system has the ability to enable or disable the interface to the mobile device at any time. When it is enabled, an icon is displayed on system's imaging screen, reminding the user that a mobile device can connect to the system.

If access needs to be denied to a user who has previously used the app, the ACUSON Freestyle MobileLink PIN code on the ultrasound system can be changed. Changing the PIN code on the ultrasound system will automatically change the GUID. On the next command sent from the mobile device to the system, the GUIDs will not match, and access will be denied.

\section{Management of Electronic Protected Health INFORMATION}

Images transferred to the app will have the patient's name and ID embedded in the image. If this is a concern, the ACUSON Freestyle system has features to anonymize the images. The features will prevent the patient's name and ID from being embedded into the saved image, while preserving this data internally for use when transferring the images in DICOM format to an image archiving system. The anonymize feature is useful for emailing of images.

\section{Regulatory}

The ACUSON Freestyle ${ }^{\mathrm{TM}}$ system was cleared by the US FDA under 510(k). It is a Class II device.

Does a mobile app that interfaces to the system require a 510(k) for US FDA clearance? The FDA has published a guidance document "Mobile Medical Applications" [7] which states that "Examples of mobile apps that display, store, or transfer medical device data in its original format include: apps that are intended to display or store medical device data, without controlling or altering the functions or parameters of any connected medical device constitute a Medical Device Data System (MDDS) (21 CFR 880.6310) and are subject to class I requirements (general controls)." Therefore, no $510(\mathrm{k})$ submission is necessary.

In a March 2014 update to the Mobile Medical App guidance, the FDA explicitly addressed apps similar to the ACUSON Freestyle MobileLink app: "Mobile apps that are not intended for diagnostic image review (and include a persistent on-screen notice, such as "for informational purposes only and not intended for diagnostic use") are medical image communications device [sic] under $21 \mathrm{CFR}$ 892.2020, product code LMB. Non-diagnostic uses could include: image display for multidisciplinary patient management meetings (e.g., rounds) or patient consultation. Medical image communications devices are Class I and do not require FDA 510(k) premarket notification. Possible product code LMD (21 CFR 892.2020).’ [8]

Since the images are transferred from the ACUSON Freestyle $^{\mathrm{TM}}$ system to the app in their original format and the app is not used to control the system, the app is classified as a Class I device, which is not subject to a $510(\mathrm{k})$ submission. The on-screen notice "For informational purposes only. Not intended for diagnostic use." is always shown on the Patient Study list. The message is also included in the default body of any email that is generated by the app when emailing image(s) from a study.

\section{ApP EVAluation AND TESTING}

The ACUSON Freestyle MobileLink app has been tested on various Windows 8.1 mobile devices, including Microsoft Surface Pro $2^{\mathrm{TM}}$, Microsoft Surface Pro $3^{\mathrm{TM}}$, Dell Venue $8^{\mathrm{TM}}$, and Lenovo ThinkPad $8^{\mathrm{TM}}$ tablets.

The initial response of physicians and clinicians who have seen the app has been positive. Comments that have been shared include: 1) Could be utilized as a teaching tool for education of physicians in training, 2) Sharing of images via email with colleagues was viewed as a strong point, 3) Useful for patient education - illustrating to the patient the anatomy that has been examined.

\section{Future DEVELOPMENT}

Future development of the app is planned. New features might include remote control of the ACUSON Freestyle ${ }^{\mathrm{TM}}$ system from the mobile device, voice control of the system using the voice recognition capability of the mobile device and real-time streaming of images from the system to the mobile device. Implementation of these features may require a $510(\mathrm{k})$ submission.

\section{REFERENCES}

[1] Siemens Medical Solutions USA, Inc. "Find your Freedom. ACUSON Freestyle ${ }^{\mathrm{TM}}$ Ultrasound System", 02.2013

[2] Siemens Medical Solutions USA, Inc, Datasheet - "ACUSON Freestyle ${ }^{\mathrm{TM}}$ Ultrasound System Release 3.0", 05.2013

[3] National Electrical Manufacturers Association Digital Imaging and Communications in Medicine (DICOM), Parts 1 - 20, 2013 Version

[4] Apple Inc, "QuickTime File Format Specification" 2013-08-14

[5] Microsoft Corporation, Microsoft Developer Network website (www.msdn.microsoft.com) "XAML Overview", retreived 2014-09-18

[6] Microsoft Corporation, Microsoft Developer Network website (www.msdn.microsoft.com) "MVVM Reference Implementation", retreived 2014-09-18

[7] U.S. Department of Health and Human Services, Food and Drug Administration, "Mobile Medical Applications Guidance for Industry and Food and Drug Administration Staff", September 25, 2013

[8] U.S. Department of Health and Human Services, Food and Drug Administration website (www.fda.gov) "Examples of MMAs the FDA Regulates", retrieved 2014-09-10 